\title{
Anspruch auf staatliche Kostenbeteiligung bei ambulanten Pflegeleistungen
}

Besprechung des Urteils des Bundesgerichts 9C_176/2016 vom

21. Februar 2017

Andreas Petrik*

Erbringt eine Pflegefachperson auf Grundlage einer ärztlichen Verordnung ambulante Pflegeleistungen, werden die Kosten zwischen Krankenversicherer, öffentlicher Hand und Leistungsempfänger aufgeteilt. Gemäss Bundesgericht kann der Beitrag des Staates zur Sicherstellung der Wirtschaftlichkeit, der Zweckmässigkeit und der Wirksamkeit der Leistungen von weiteren Voraussetzungen abhängig gemacht werden. Dass der Krankenversicherer seine Leistungspflicht anerkennt, bedeutet nicht, dass auch der Staat seinen Anteil vergüten muss.

I. Sachverhalt 105

II. Erwägungen 105

III. Bemerkungen 106

Zitiervorschlag: Andreas Petrik, Anspruch auf staatliche Kostenbeteiligung bei ambulanten Pflegeleistungen, in: sui-generis 2018, S. 104

URL: $\quad$ sui-generis.ch/61

DOI: $\quad$ https://doi.org/10.21257/sg.61

* Andreas Petrik ist als Rechtsanwalt in St.Gallen und Winterthur tätig. Als Vertrauensanwalt der Sektion St.Gallen/Thurgau/Appenzell des Schweizer Berufsverbands der Pflegefachfrauen und Pflegefachmänner (SBK) vertritt er regelmässig Verbandsmitglieder in arbeits-, sozialversicherungs- und strafrechtlichen Angelegenheiten. In der Angelegenheit, die das Bundesgericht im hier besprochenen Urteil entschied, vertrat der Autor die Beschwerdeführerin vor Bundesgericht. 


\section{Sachverhalt}

1 A. erbringt in verschiedenen Gemeinden im Kanton Thurgau als selbständig Erwerbende Leistungen im Bereich der ambulanten Krankenpflege. Darunter fallen unter anderem das Einüben von Bewältigungsstrategien, die Anleitung im Umgang mit Aggression, Angst und Wahnvorstellungen sowie die Unterstützung für psychisch kranke Personen in Krisensituationen, insbesondere zur Vermeidung von akuter Selbst- oder Fremdgefährdung.

2 Eine teilweise Kostenübernahme durch die Krankenversicherer und die öffentliche Hand - ein weiterer Teil der Kosten wird vom Leistungsempfänger getragen - solcher Pflegeleistungen setzt unter anderem voraus, dass der Pflegebedarf auf Grundlage einer ärztlichen Verordnung von einer zur Bedarfserfassung zugelassenen Person erfasst wurde und die Pflegeleistungen vom zuständigen Krankenversicherer als wirksam, zweckmässig und wirtschaftlich angesehen werden.

3 Die von A. erbrachten Pflegeleistungen basierten auf ärztlichen Verordnungen und wurden von den Krankenversicherern übernommen. Die Gemeinde, die gemäss kantonalem Recht für die Bezahlung der sogenannten Restkosten - also jenen Kosten die weder vom Krankenversicherer noch vom Leistungsempfänger getragen werden - zuständig war, verweigerte die Kostenübernahme mit dem Hinweis auf die kantonale Verordnung, wonach die Kosten nur dann übernommen werden, wenn ein bestimmtes Bedarfserfassungssystem verwendet wird.
4 Der Gemeinderat, das Department für Finanzen und Soziales des Kantons Thurgau und auch das kantonale Verwaltungsgericht befanden, dass A. keinen Anspruch auf die Abgeltung der Restkosten habe, da sie nicht das in der Verordnung vorgesehene Bedarfserfassungssystem verwende. Gegen das Urteil des kantonalen Verwaltungsgerichts erhob A. Beschwerde beim Bundesgericht. Das Bundesgericht wies die Beschwerde ab.

\section{Erwägungen}

5 Das Bundesgericht hatte sich in erster Linie mit der Frage auseinanderzusetzen, ob der Anspruch der Beschwerdeführerin auf Bezahlung der Restkosten durch die Gemeinde abschliessend im Bundesrecht geregelt ist oder ob die Kantone weitere Voraussetzungen für die Übernahme der Restkosten vorsehen können. Gemäss den Ausführungen des Bundesgerichts sei die Rechtsprechung, wonach von den Kantonen zugelassene Leistungserbringer einen bundesrechtlichen Anspruch auf Abgeltung der Restkosten haben, im Zusammenhang mit der ambulanten Krankenpflege nicht einschlägig. Diese Rechtsprechung beziehe sich ausschliesslich auf die Restkostenübernahme von Patienten in stationären Einrichtungen. In Bezug auf die Restkostenabgeltung im Bereich der ambulanten Krankenpflege rechtfertige es sich deshalb, den Anspruch zur Wahrung der Wirksamkeit, Zweckmässigkeit und der Wirtschaftlichkeit von weiteren Voraussetzungen abhängig zu machen. Ob der öffentlichen Hand darüber hinaus auch die Kompetenz zukommt, die Einhaltung der genannten Kriterien im Einzelfall zu prüfen, liess das Bundesgericht offen. 
$6 \mathrm{Zu}$ entscheiden hatte das Bundesgericht weiter darüber, ob sich $\S 41$ der thurgauischen Verordnung des Regierungsrates zum Gesetz über die Krankenversicherung auf eine hinreichende gesetzliche Grundlage stützt. Das Bundesgericht bejahte diese Frage und verwies zur Begründung auf die Bestimmungen des kantonalen Gesetzes über die Krankenversicherung.

7 Schliesslich stellte das Bundesgericht fest, dass das von der Beschwerdeführerin verwendete Bedarfserfassungssystem den gesetzlichen Anforderungen nicht genüge, da die Bedarfserfassung nicht auf einheitlichen Kriterien beruhe. Das System würde keine ausreichend strukturierte und standardisierte Erfassung ermöglichen. Ausserdem erbringe die Beschwerdeführerin nicht nur psychiatrische Pflegeleistungen sondern auch somatische, wobei das von ihr verwendete Erfassungssystem lediglich auf die pflegerische Bedarfsabklärung bei Personen mit psychischen Einschränkungen zugeschnitten sei.

\section{Bemerkungen}

Das Bundesgericht hatte sich bereits früher mit der Frage auseinanderzusetzen, ob der Anspruch auf Abgeltung der Restkosten durch die öffentliche Hand abschliessend durch das Bundesrecht geregelt ist oder ob die Kantone die Abgeltung dieser durch den Staat zu tragenden Pflegekosten von weiteren Bedingungen abhängig machen können. Gemäss Art. 25a KVG dürfen der versicherten Person von den nicht von Sozialversicherungen gedeckten Pflegekosten höchstens 20 Prozent des höchsten vom Bundesrat festgesetzten Pflegebeitrages überwälzt werden. Die Regelung der Restfinanzierung wird den Kantonen übertragen. Im Zusammenhang mit der Abgeltung der Restkosten bei stationärer Pflege hat das Bundesgericht bereits mehrfach (bspw. BGE 138 II 191 und BGE 138 I 141) festgehalten, dass Art. 25a Abs. 5 KVG den Kantonen vorschreibe, diese Kosten bei den Leistungserbringern ohne weitere Bedingungen zu decken, die ermächtigt sind, ihre Leistungen der obligatorischen Krankenversicherung zu berechnen. Ausserhalb der bundesrechtlichen Regelung der Restkostenabgeltung bestehe für die Kantone jedoch weiterhin die Möglichkeit, die Ausrichtung von Subventionen von weiteren Bedingungen abhängig zu machen.

9 Davon abweichend erachtet das Bundesgericht im hier besprochenen Entscheid in Erwägung 6.2.1 die kantonale Regelung, wonach lediglich dann Anspruch auf Abgeltung der Restkosten bestehe, wenn ein bestimmtes Bedarfserfassungssystem verwendet werde, als mit den bundesrechtlichen Vorgaben vereinbar. Zur Begründung wird ausgeführt, dass sich die bisherige Rechtsprechung ausschliesslich auf die Restkostenabgeltung bei stationärer Pflege beziehe und diese naturgemäss einer höheren Regelungsund Kontrolldichte unterliegen würde, als dies bei freiberuflich tätigen Pflegefachpersonen der Fall sei. Zur Wahrung der Wirksamkeit, der Zweckmässigkeit und der Wirtschaftlichkeit der erbrachten Pflegeleistungen sei es den Kantonen im Bereich der ambulanten Pflege erlaubt, die Abgeltung der Restkosten von der Verwendung eines bestimmten Systems der Bedarfserfassung abhängig zu machen. 
10 Gemäss dem Entscheid des Bundesgerichts haben die Kantone also neuerdings nicht mehr nur die Möglichkeit, die Voraussetzungen für die Zulassung von freiberuflich tätigen Pflegefachpersonen und für die Ausrichtung von Subventionen zu regeln, sondern sie können auch den Anspruch auf Abgeltung der durch das Bundesrecht geregelten Übernahme der Restkosten von weiteren Erfordernissen abhängig machen. Ob dies - wie vom Bundesgericht ausgeführt - zur Sicherstellung der Wirksamkeit, der Zweckmässigkeit und der Wirtschaftlichkeit der erbrachten Pflegeleistungen tatsächlich notwendig ist, erscheint zumindest fraglich, da den Krankenversicherern diesbezüglich eine umfassende Prüfungskompetenz zukommt und diese von Gesetzes wegen ausschliesslich Leistungen übernehmen dürfen, die wirksam, zweckmässig und wirtschaftlich sind.

11 Um die Rechtmässigkeit der Kostenübernahme durch die Krankenversicherer sicherzustellen, haben die Leistungserbringer und die Krankenversicherer auf Grundlage der KrankenpflegeLeistungsverordnung (KLV) das Verfahren zur Überprüfung der Bedarfsabklärung und zur Kontrolle der Zweckmässigkeit und Wirtschaftlichkeit der Leistungen eingehend geregelt. Das Kontrollverfahren ist im Administrativvertrag zwischen dem Schweizer Berufsverband der Pflegefachfrauen und Pflegefachmänner (SBK) und den Krankenversicherern (AdminV) festgelegt. Die Versicherer prüfen die Gesetzmässigkeit der Leistungen zunächst anhand des Bedarfsmeldeformulars und des Rechnungsformulars. Bei Bedarf können auch weitere Unterlagen eingefordert werden. Weiter werden die Leistungen durch Kontrollbe-

suche bei den Leistungserbringern geprüft. Diese haben Einsicht in die Patientendossiers zu gewähren. Schliesslich erfolgt eine Kontrolle auch aufgrund einer Einzelfallprüfung beim Versicherten.

12 der Versicherer, die Leistungen auf ihre Wirtschaftlichkeit zu überprüfen, und der weitgehenden Möglichkeiten, wie sie im AdminV festgelegt sind, macht es kaum Sinn, wenn das Bundesgericht den Kantonen die Kompetenz einräumt, die Wirtschaftlichkeit, die Zweckmässigkeit und die Wirksamkeit zusätzlich durch gesetzliche Vorkehren sicherzustellen.

13 Ob darüber hinaus auch die Kompetenz zur Einzelfallprüfung besteht, lässt das Bundesgericht offen, und schliesst damit die Zulässigkeit von entsprechenden Massnahmen zumindest nicht explizit aus. Sollte ein Kanton aufgrund der Erwägungen des Bundesgerichts tatsächlich auf die Idee kommen, ambulante Pflegeleistungen auf ihre Gesetzmässigkeit zu überprüfen, würde sich eine Reihe von Fragen stellen. Zunächst müsste sichergestellt werden, dass das notwendige Fachwissen verfügbar ist, das für eine Prüfung erforderlich wäre. Sodann würden sich auch datenschutzrechtliche Fragen stellen. Während die Krankenversicherer den entsprechenden Regelungen des Krankenversicherungsgesetzes unterstellt sind, ergäben sich bei einer zusätzlichen Prüfung der Leistungspflicht der öffentlichen Hand Fragen hinsichtlich der Sicherstellung des Datenschutzes. So ist bespielweise die Möglichkeit einer vertrauensärztlichen Untersuchung auf Anordnung der Krankenversicherung gesetzlich geregelt, während die Rah- 
menbedingungen für eine entsprechende Anordnung durch den Staat nicht geregelt sind. Schliesslich kann nicht ausgeschlossen werden, dass sich bei gleichzeitiger Prüfung durch den Staat und die Krankenversicherer unterschiedliche Resultate ergeben.

14 Wie das Urteil des Bundesgerichts zeigt, führen auch kantonale Regelungen, die die Abgeltung von Restkosten an weitere Voraussetzungen knüpfen, dazu, dass Leistungen, die von der Krankenversicherung übernommen werden, nicht in jedem Fall vom Staat mitfinanziert werden müssen. Ein solches Ergebnis erscheint nicht sachgerecht. Konsequenter wäre es, wenn die Kantone zwar die $\mathrm{Zu}$ lassung der Leistungserbringer und die Ausrichtung von allfälligen Subventionen regeln können, zur Sicherstellung der Wirtschaftlichkeit, Zweckmässigkeit und Wirksamkeit der Leistungen jedoch keine - über die Regelungen des KVG - hinausgehenden Bedingungen vorsehen dürften. 